Moreover, it was observed that the standard itself had acquired some tarnish. Some say that this was hastened by workers with the small weights, who purposely heated some vitriol because they. had tired of being on the negative end of things, but be that as it may, it was only the inevitable that happened when the standard was finally removed from its primary status.

In order to preserve the units in general use it was decided to adopt only the unexcelled lighter stone as a standard of 273 convenient units and soon everyone wondered why they had done otherwise.

Explanatory Note. It has not been expedient to simulate more closely the actual historical sequences and facts in making a point that refers to a twentieth century situation. As a matter of fact, there was an excellent reason for two easily obtainable fixed points in the early days of thermometry.

In 1654, Grand Duke Ferdinand II of Tuscany had bulb and stem thermometers calibrated in thousandth parts of the bulb volume. This very sensible approach to the problem, which required only one fixed point, was discarded later only because the expansion of various liquids did not yield identical results. This led to two fixed points with the scale interval divided into a certain number of divisions and brought various thermometers into somewhat better yet crude agreement.

In 1701 Newton proposed the interval between the ice point and the none too uniform temperature of the human body as a scale of 12 degrees, and in 1714 Fahrenheit substituted the ammonium chloride - ice eutectic temperature for the ice point to avoid negative temperatures; but unfortunately the stage had not been set sufficiently so that he could see the ultimate goal. Later, Fahrenheit investigated and adopted the present fixed points. The centigrade scale was not proposed until 1742, when Celsius suggested it in reverse form.

The discovery and investigation of numerous gases took place in the eighteenth and nineteenth centuries. If this kind of investigation had not been delayed for so long, the first thermometer, based on the expansion of air and invented by Galileo in 1592 , would have developed sooner into the modern gas thermometer, and it is difficult to imagine that the systems based on two fixed points would ever have originated.

${ }^{1}$ Kelvin, Proc. Camb. Phil. Soc., 1, 66 (1848); Phil. Mag., (3), 33, 313 (1848); Trans. Roy. Soc. Edinburgh, March 1851 and May 1854; Phil. Mag., (4), (1852). "Mathematical and Physical Papers, Thomson (Kelvin)", 1, 235 (Cambridge Univ. Press, 1882).

\title{
Molecular Layers
}

$\mathrm{T}$ HE foundation of the Pilgrim Trust series of lectures, of which the first was delivered by Dr. Irving Langmuir before the Royal Society in the theatre of the Royal Institution on December 8, 1938, was noted in Nature of December 17 last. In this lecture, the subject-matter of which is printed in extenso in the Proceedings of the Royal Society, A, 170, 1 (1939), he gave a comprehensive account of his investigations on monolayers and multiple layers of complex organic molecules. These researches, initiated by him in 1917, and carried on by many workers, Langmuir himself always taking a leading part in advancing both theory and experimental technique, show no sign of ceasing to be a fertile field for investigation.

The orientation of organic molecules, with a water-soluble group at one end of a long hydrocarbon chain, at a water surface with the chains perpendicular, or nearly so, to the surface, now forms part of the subject-matter of many advanced text-books. The molecules orient themselves in this manner only if the hydrocarbon chains are sufficiently long for the lateral adhesion between them to control the thermal motions of the molecules. If either the temperature is raised, or the length of the hydrocarbon chains is diminished, or the amount of adhesion between the adjacent molecules in the surface film decreases from some other change in constitution of the molecules, this simple orientation of the molecules may be destroyed. If the lateral adhesion is completely overcome, the molecules separate completely, the film becoming 'gaseous'. In the 'gaseous' state of the films, the molecules lie flat on the surface, provided there is sufficient space, and the relation between the area and the surface pressure is similar to that between volume and pressure in a gas.

There is an intermediate stage (the liquidexpanded films) between the condensed films, with molecules nearly upright, and the 'gaseous', in which they lie flat, which presented some very puzzling features until in 1933 Langmuir showed that they simulate very closely the behaviour of thin layers of a non-spreading paraffin oil, in which a small amount of a spreading substance such as a fatty acid is dissolved, thus forming a thin liquid layer of paraffin separated from the water by an 
interface containing a gaseous film of fatty acid. In the liquid-expanded films, the hydrocarbon chains in the molecule appear to be in chaotic oscillation, similar to that of the molecules in a liquid except that the water-soluble end is anchored to the water. The greater part of the cohesion of such films is provided by the adhesion between the hydrocarbon chains, which persists just as in a liquid, in spite of the chaotic motions of these chains.

During the last four years, there have been important experimental developments. It has been shown that the properties of the monolayers of fatty acids may depend on the ions with which the end groups are associated, and that this may serve as a very delicate test for minute amounts of divalent or trivalent ions in the underlying solution; as little as one ion of aluminium for thirty atoms of stearic acid may alter noticeably the properties of the material skimmed up off a surface covered by a film, this quantity corresponding to a concentration of as little as one part in three thousand million of water. As a microchemical technique, the effects of ions on monolayers appear very promising, the amount of silver or gold dissolved when a piece of the pure metal is simply dipped into water sufficing to alter the viscosity of a monolayer of stearic acid appreciably, in a few minutes. This recalls the mysterious 'oligodynamic' poisonous action on living cells, caused by contact of these metals with water, and may be a first step to the understanding of this action.

Langmuir and Blodgett's multiple layers built up by repeated dipping of a solid plate into water covered by a monolayer under constant surface pressure are perhaps the most important recent development. These layers may be built up to several thousands of molecules thick, if dipping is continued long enough; their structure seems to be very similar to that of crystals of long-chain aliphatic substances with polar groups at one end of the molecule ; that is, they have sheets of molecules parallel to the solid surface, with the molecules in successive sheets arranged with the polar groups pointing alternately towards, and away from, the solid. The layers with the polar groups directed to the solid are called 'endotropic'; those with these groups directed outwards, 'exotropic'. There are differences in the manner of deposition of these layers, according to the acidity of the water, and the salts contained in it ; if the solution under the monolayer has a $p \mathrm{H}$ greater than about 9, layers are deposited only on entering the water ( $X$ films); on slightly less alkaline solutions the layers are deposited both on entering and on leaving the water ( $Y$ films). Multiple layers are not easily deposited unless there is some calcium or barium in the solution, and they consist of a mixture of the free acid and the alkaline earth soap.

The free fatty acid may be dissolved out of the films by treatment with benzene, leaving the thickness of the film unchanged, but its refractive index is much diminished. It is possible to eliminate the reflection of light from glass, by covering it on both sides with a multiple film having a refractive index equal to the square root of that of the glass, and an optical thickness equal to one quarter wave-length; if this is done to any instrument having a glass front, the glass becomes invisible and the instrument can be used with light reflected at any angle, without disturbance from the 'shine' on the glass front.

There is some evidence that the molecules in the multiple layers can turn over. Drops of water on the surface can apparently, in some circumstances, cause the molecules to turn with their polar groups outwards. Dipping into solutions of such salts as thorium nitrate turns the exterior molecules over, and such 'conditioned' surfaces have remarkably interesting powers of adsorption ; they are hydrophilic instead of hydrophobic, as the exterior 'endotropic' layers normally are. Adsorption of bile acids on such conditioned surfaces confers hydrophobic properties on the surfaces, but not of exactly the same kind as the properties of the original unconditioned outer layer. For some reason which is not yet clear, the surface of the unconditioned films, although hydrophobic, forms a finite angle of contact with oil and does not easily adsorb paraffin vapours. A conditioned layer on which a bile acid is adsorbed can in its turn adsorb hydrocarbon vapours, thus showing that adsorption even in simple cases depends on the detailed molecular architecture of the surface. Quite small stereochemical differences, for example that between cholesterol and epicholesterol, make a great difference between the power of penetration of digitonin into the outer layers of deposited films.

Monolayers of proteins are another field in which opportunities seem abundant. In solution, the proteins are compact molecules; on surfaces, they are unfolded to a thickness of probably one amino-acid. This may account for the denaturation of the protein, since the re-folding of a very complicated molecule, once it has been spread on a surface, is likely to be difficult. Some preliminary work on enzymes indicates some loss of activity when they are spread at a surface.

The lecture contains not only a survey of the whole of the work on molecular layers, but also it is full of most valuable new hints on technique, and suggests several new fields for further research. N. K. A. 